Original Paper

في التراق عن وجود تغيرات في الفطر . Tilletia spp المسبب لمرض التفحم المغطى (البنط) مكارم محمد بشير يونس، حسن يوسف جابر، نبيل نوري محمد علي، اياد حسن كاظم، حسن عبد الواحد عباس، سحر نعيم

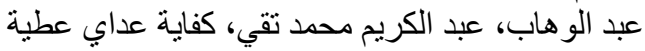

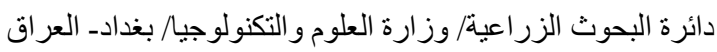

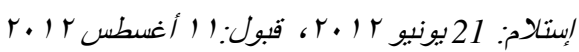

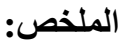

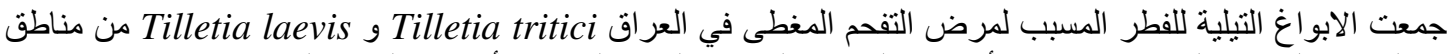

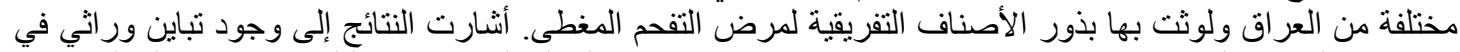

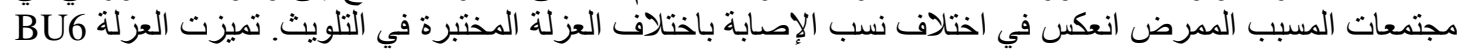

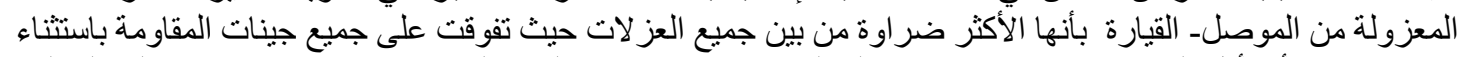

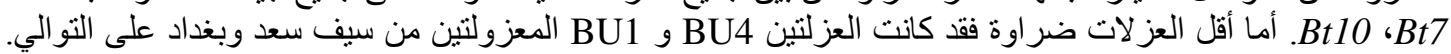

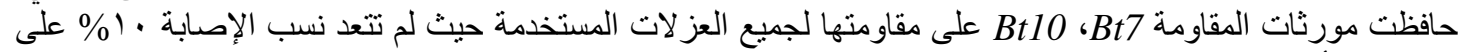

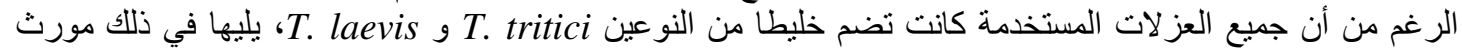

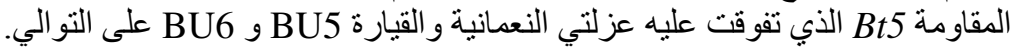

الكلمات المفتاحية: فطريات التفحم المغطى، الجينات المقاومة، التباين الوراثي.

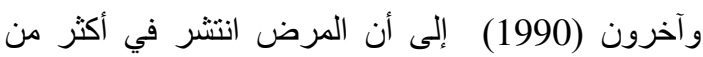

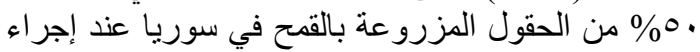

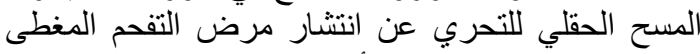

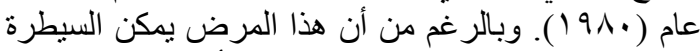

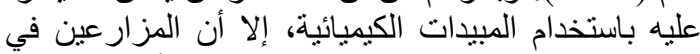

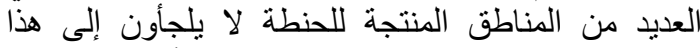

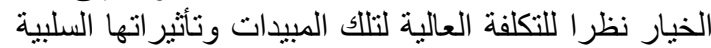

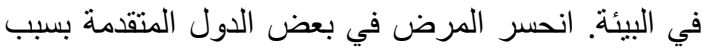

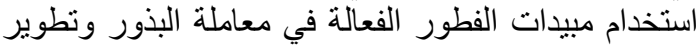
أصناف مقاومة (Goates،

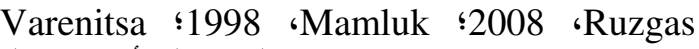
وجماعته، 1987). إلا إنه لا زال يشكل أحد عوامل

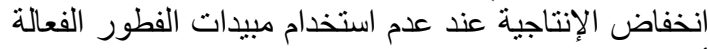

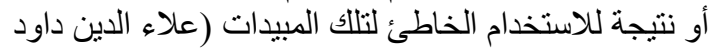

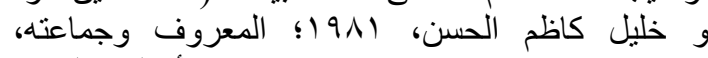
990 1990 Al-Baldawi، 1993). إن إنباع أساليب التربية

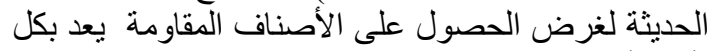

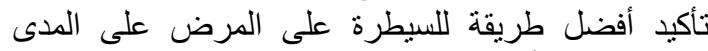

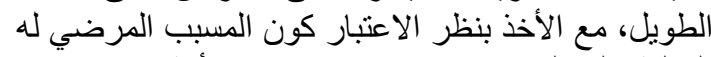

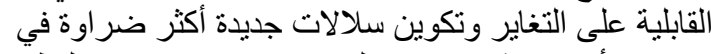
بعض الأحيان. شخصت عالمير ونكيا عدة سلالات من الفطر

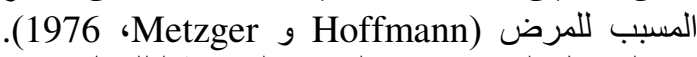
وتم التوصل إلى مستوى عالي من المقاومة لتلألك السلالات

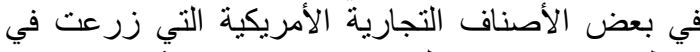

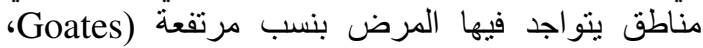
1994؛ Souza، 1995). لوحظ حدوث مستويات عالية

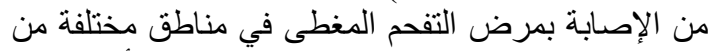

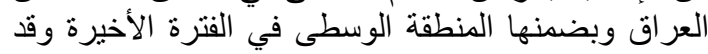

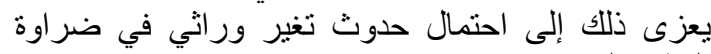

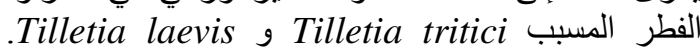
هدف البحث الكذكور إلى التحري عن وجود ذلاتك التغير من

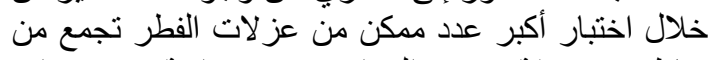

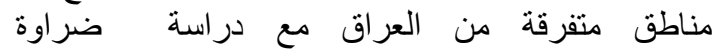

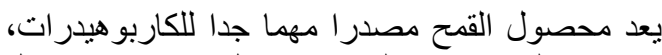

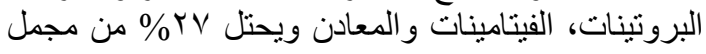

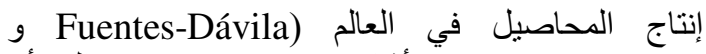
Rajaram

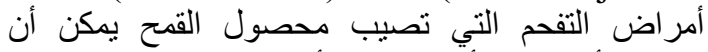
تضاهي أمر اض الأصداء في الأهمية الاقتصادية من حيث

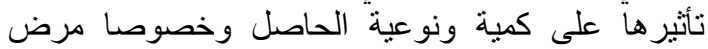

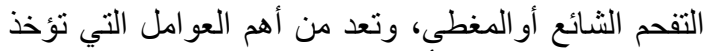
بنظر الاعتبار في أنظمة شهادات التصدية التصديق المحلية

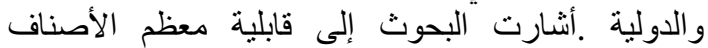
العراقية المزروعة للإصابة بأمر اض التفحمات (المعروف

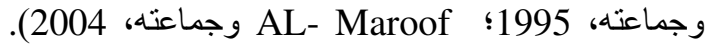

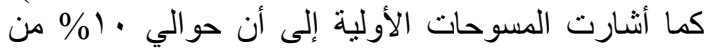

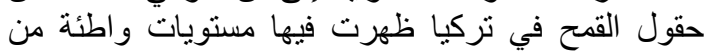

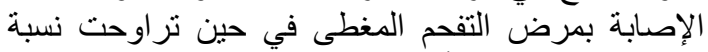

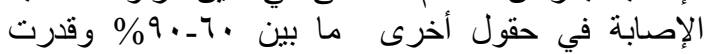

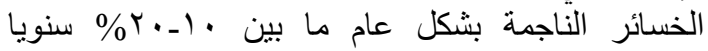

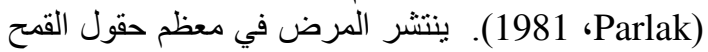

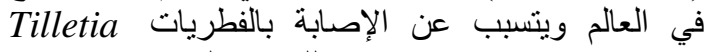

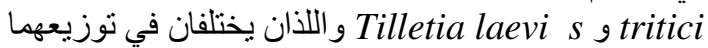
الجغرافي ومداهما العائلي وكذللك في الثنكل المظهري

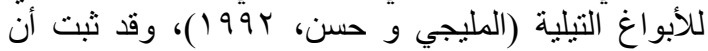

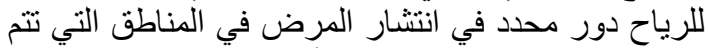

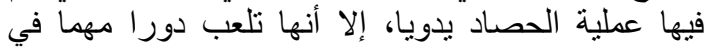

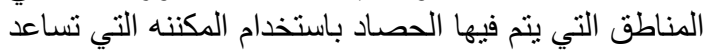

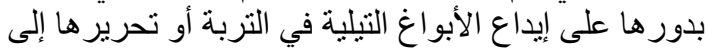

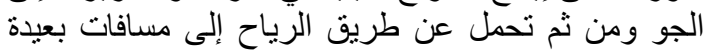

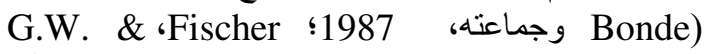

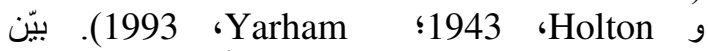
المakluk) المغطى له أهية كبيرة في إصابة محصول القمح في أكثر Mamluk مناطق دول شرق أسيا وشمال أفريقيا وأضاف في الفير 


\begin{tabular}{|c|c|}
\hline $\begin{array}{c}\text { مورثات المقاومة } \\
\text { Resistant Genes }\end{array}$ & $\begin{array}{c}\text { الأصناف التفريقية } \\
\text { Differential Varieties } \\
\text { '(أصناف فمح ربيعية) }\end{array}$ \\
\hline$B t-1$ & $\begin{array}{c}\text { M84-512 to } 520, \mathrm{RB} / \mathrm{WF} \\
38 .\end{array}$ \\
\hline$B t-2$ & $\begin{array}{l}\text { M84-522 to 530, } \\
\text { RB/SEL } 1403\end{array}$ \\
\hline$B t-3$ & $\begin{array}{l}\text { M84-532 to 538, } \\
\text { RB/RDT. }\end{array}$ \\
\hline$B t-4$ & $\begin{array}{l}\text { M82-542 to } 550, \\
\text { RB/TK } 3055\end{array}$ \\
\hline$B t-5$ & Red Bobs/Hohenheimer \\
\hline$B t-7$ & $\begin{array}{c}\text { M84-562 to } 570, \mathrm{RB} / \mathrm{TK} \\
3055\end{array}$ \\
\hline$B t-8$ & $\begin{array}{c}\text { M78-9496, RB/PI } \\
178210 \text { (White Seeds) }\end{array}$ \\
\hline$B t-10$ & $\begin{array}{c}\text { M84-625, SEL. M83- } \\
162 .\end{array}$ \\
\hline$B t-14$ & Doubi, DW \\
\hline$B t-15$ & Carlton, DW \\
\hline
\end{tabular}

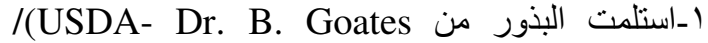
أبردين/ ولاية أيداهو الأمريكية للموسم V.... ARS)

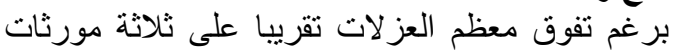

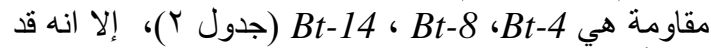

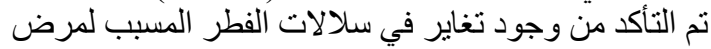

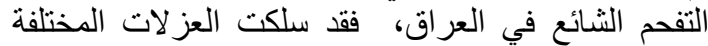

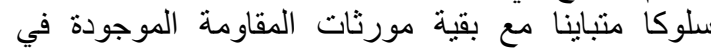

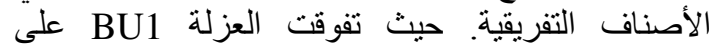

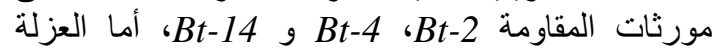

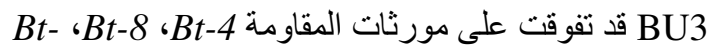

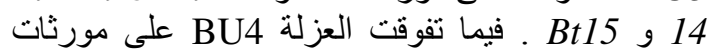

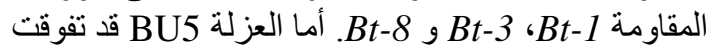

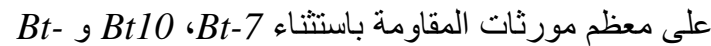

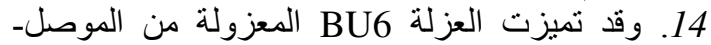

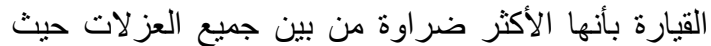

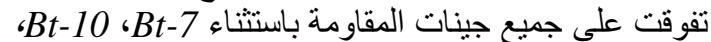

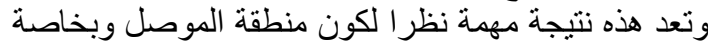

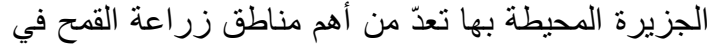

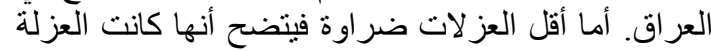

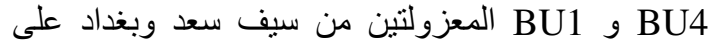

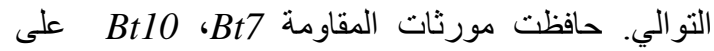

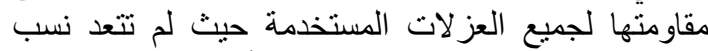

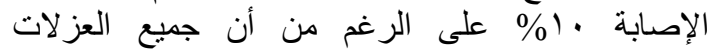

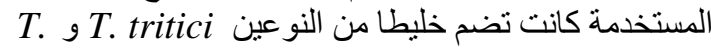
laevis

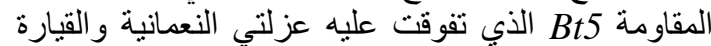
فقط. BU6 و BU5
في المجتمع السكاني الموجود في العراق (Virulence) من خلال دراسة التخصص الفسلجي (استخدام الأصناف التفريقية) للتحري عن وجود تغايرات في مجتمع المسبب الإنبان المرضي. مواد البحث وطرائقه: - ماته

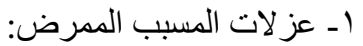

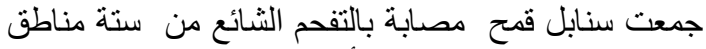

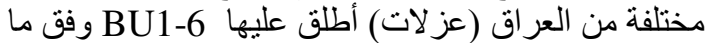

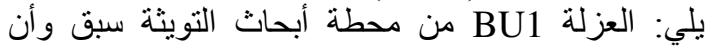

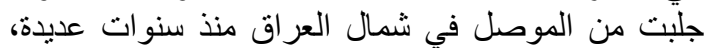

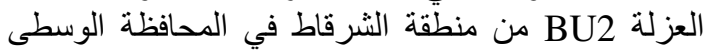
صلاح الدين، العزلة BU3 من من منطقة جلو لكن لاء في محافظة

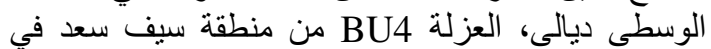

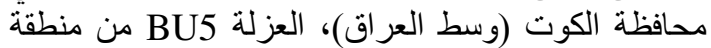
النعمانية جنوب العراق، العزلة BU6 من منطقة القيارة جنوب الموصل. استخلصت الأبواغ التبلية من الكرات الكرات التفحمية لكل منطقة على حدة ثم حفظت في علب زجاجية لإنية مُعتمة لحين استخدامها لاحقا.

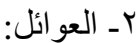

استخدمت في هذه الدراسة أصناف التمبيز الدولية وتحديدا الأصناف الربيعية (Differential Varieties)

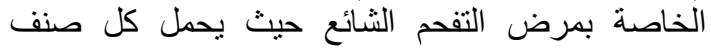

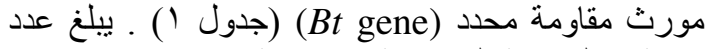

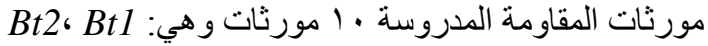
Bt15 B14 Bt10، Bt8 ، Bt7، Bt5 ، Bt4 ، Bt3،

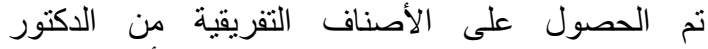
B.Goates إيداهو الأمريكية).

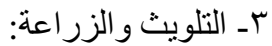
لوثت بذور جميع أصناف التفريق الدولية التي تحمل

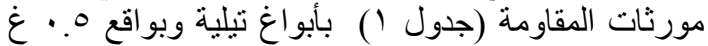

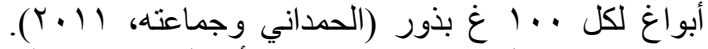

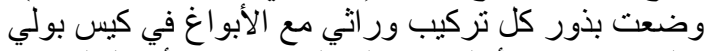

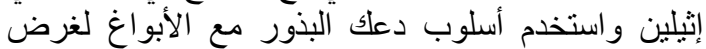

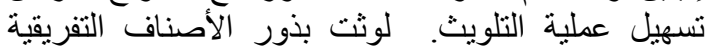
بأبواغ جميع العزلات الستة. أجريت التجارب لون في محطة التونة

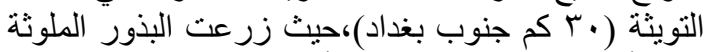

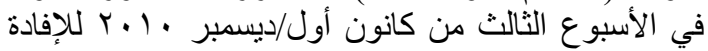

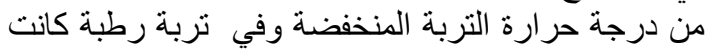

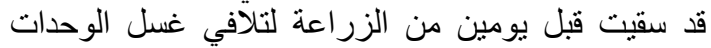

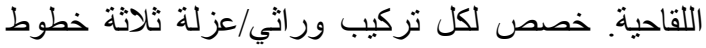

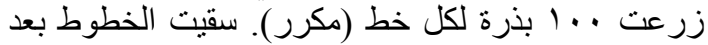

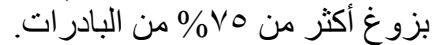

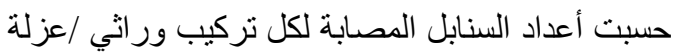
في مرحلة النضج واستخرجت النسب المئوية للإصابة. استخدم في تحديد أنماط الإصابة (Infection Types)

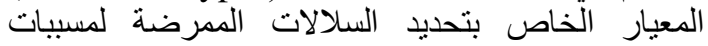

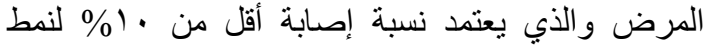
الإصابة المنخفض (Low Infection Type) أي المقاوم

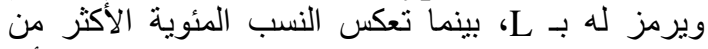

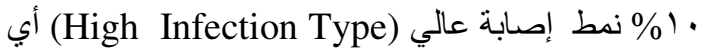

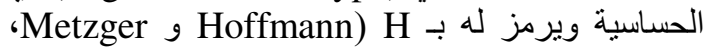

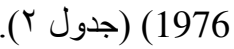




\begin{tabular}{|c|c|c|c|c|c|c|}
\hline \multicolumn{6}{|c|}{ النسبة المئوية للإصابة بعز لات المسبب الممرض } & \multirow{3}{*}{ 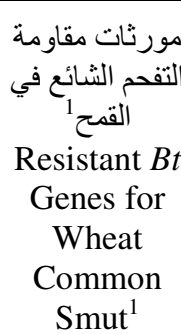 } \\
\hline BU6 & BU5 & BU4 & BU3 & BU2 & BU1 & \\
\hline القيارة & النعمانية & سيف سعد & جلو لاء & الثرقاط & بغداد & \\
\hline $\mathrm{H}$ & $\mathrm{H}$ & $\mathrm{H}$ & $\mathrm{L}$ & $\mathrm{H}$ & $\mathrm{L}^{3}$ & $B t-1$ \\
\hline $\mathrm{H}$ & $\mathrm{H}$ & $\mathrm{L}$ & $\mathrm{L}$ & $\mathrm{L}$ & $\mathrm{H}$ & $B t-2$ \\
\hline $\mathrm{H}$ & $\mathrm{H}$ & $\mathrm{H}$ & $\mathrm{L}$ & $\mathrm{L}$ & $\mathrm{L}$ & $B t-3$ \\
\hline $\mathrm{H}$ & $\mathrm{H}$ & $\mathrm{L}$ & $\mathrm{H}$ & $\mathrm{H}$ & $\mathrm{H}$ & $B t-4$ \\
\hline $\mathrm{H}$ & $\mathrm{H}$ & $\mathrm{L}$ & $\mathrm{L}$ & $\mathrm{L}$ & $\mathrm{L}$ & $B t-5$ \\
\hline $\mathrm{L}$ & $\mathrm{L}$ & $\mathrm{L}$ & $\mathrm{L}$ & $\mathrm{L}$ & $\mathrm{L}$ & $B t-7$ \\
\hline $\mathrm{H}$ & $\mathrm{H}$ & $\mathrm{H}$ & $\mathrm{H}$ & $\mathrm{H}$ & $\begin{array}{l}\mathrm{L} \\
\end{array}$ & $B t-8$ \\
\hline $\mathrm{L}$ & $\mathrm{L}$ & $\mathrm{L}$ & $\mathrm{L}$ & $\mathrm{L}$ & $\mathrm{L}$ & $B t-10$ \\
\hline $\mathrm{H}$ & $\mathrm{L}$ & $\mathrm{L}$ & $\mathrm{H}$ & $\mathrm{H}$ & $\mathrm{H}$ & Bt-14 \\
\hline $\mathrm{H}$ & $\mathrm{H}$ & L & $\mathrm{H}$ & $\mathrm{H}$ & $\mathrm{L}$ & $B t-15$ \\
\hline
\end{tabular}

جدول (r). أنماط إصابة ـ من أصناف التففيق الدولية وفق المعيارين (L) و (H) ) بعد تلويثها اصطناعيا بستة عز لات من المسبب

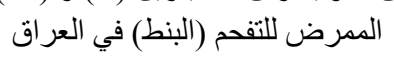

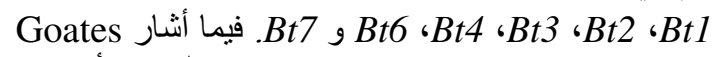

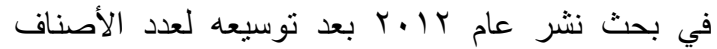

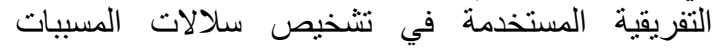

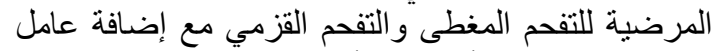

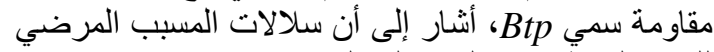

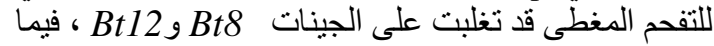

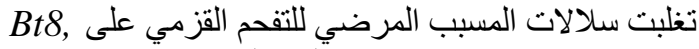
Bt11 Bt12 و على خليط من Bt19, Bt10, Bt11, Bt12

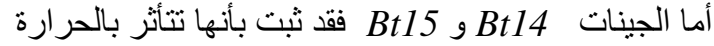
لذا استبعدت من المجمو عة لتجنب الإرباك و النتائج الخاطئنة في تحديد التفاعلات المرضية للمسببات المرضية المراكية

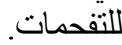

تمثل دراسات مجتمع مرض التفحم الثائع والسلالات

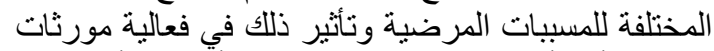

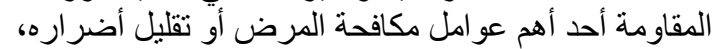

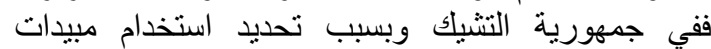
الفطور في تعفير بذور القمح، إضافة إلى الأخطاء

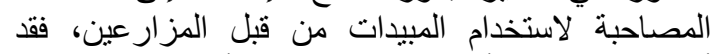

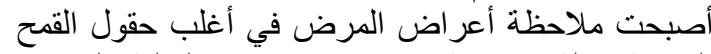

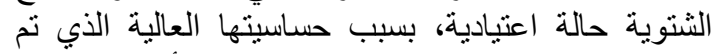
إثباتها من خلال تلويث بذور هادية بع خليط من أبو اغ النوعين .T. laevis و T. tritici

وباستخدام تقنية الواسمات الجزيئية (Molecular (markers

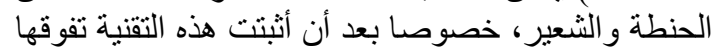

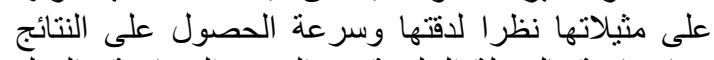

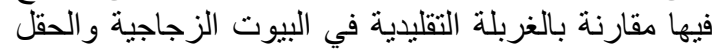
و التي تستهلاك الكثير من الوقت و الجهـ و التكلفة. ومن خلال الكثير من البحوث و الدراسات العالمية في هذا الدجال فقد العيد

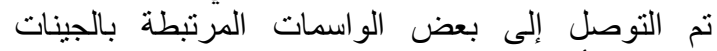
المقاومة لأمر اض التفحمات ومنها مرض الته التفحم المغطئ.

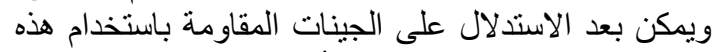

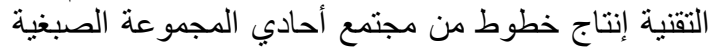

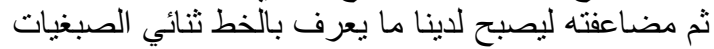
Doubled haploid

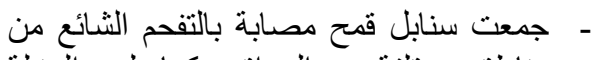
مناطق مختلفة من العراق وكما يلي: العزلة العنة

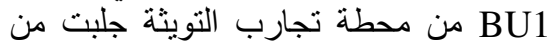

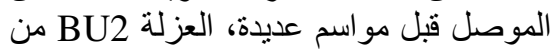

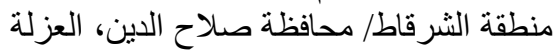

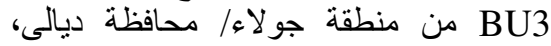
العزلة BU4 من منطقة سيف سعد/ محافظة منة منية الكوت، العزلة BU5 من من منطقة النعة النعمانية جنوب العراق، العزلة BU6 من من منطقة

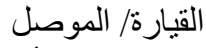
r- الستخدمت الأصناف الربيعية في مجموعة الأصناف التفريقية فقط.

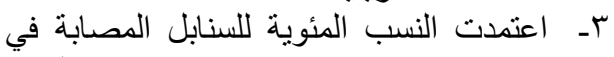

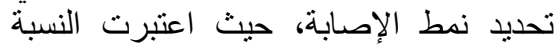

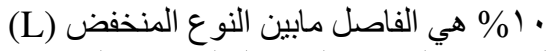

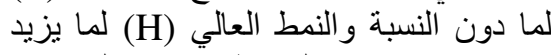
عن · (\% استتادا إلى نظام تحديد السلالات T. المرضية للفطرين المسببين للمرضم و Hoffmann) T. laevis g tritici (1976،Metzger

لم تتمكن اي عزلة من العزلات الإلة المدروسة من إحداث

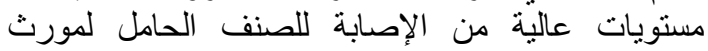
المقاومة Bt7 و هذه النتيجة تنطابق مع نتائج در اسة سابقة لإنة

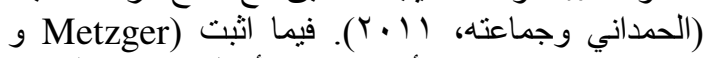
1978 Hoffman

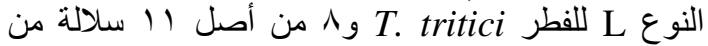

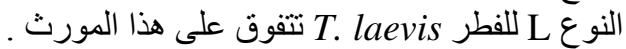

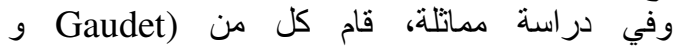
1989 Puchalski

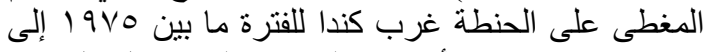
إجـ19 ا، وباستخدام الأصناف التفريقية التي يحمل كل منها

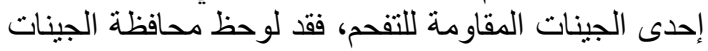

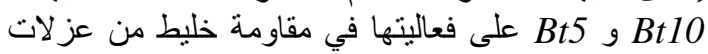
T. foetida و T. caries النتائج، بينما تغلبت هذه العزلات على مقاومة الجينات 
caries and $\mathrm{T}$. foetida) of wheat in western Canada. Canadian Journal of Plant Pathology. Volume 11, Issue 4, 1989

Goates, B.J. (2012). Identification of New Pathogenic Races of Common Bunt and Dwarf Bunt Fungi, and Evaluation of Known Races Using an Expanded Set of Differential Wheat Lines. Plant Disease, March 2012, Volume 96, Number 3, Pages 361-369

Goates, B.J. (1996). Common bunt and dwarf bunt. Pages 12-25 In: Wilcoxon, R. D.and E. E. Saari, (eds): Bunt and Smut Diseases of Wheat: Concept and Methods of Disease Management. CIMMYT, Mexico, D.F.

Goates, B.J. (1994). Resistance to dwarf bunt among winter wheat cultivars. Phytopathology, 84: 1116.

Fuentes-Dávila, G. \& Rajaram, S. (1994). Sources of resistance to Tilletia indica in wheat (Triticum aestivum). Crop Prot., 13: 20-24.

Hoffmann, J.A. \& Metzger, R.J. (1976). Current status of virulence genes and pathogenic races of the wheat bunt fungi in the northwestern USA. Phytopathology, 66: 657-660.

Liatukas, Z. and Ruzgas, V. (2008). Resistance genes and sources for control of wheat common bunt Tilletia tritici (DC.) Tul. Biologija, 54(4): 274-278.

Mamluk, O.F. (1998). Bunt and smut of wheat in North Africa and Near East. Euphytica, $100 \quad$ (1-3):45-50. Mozgovoi, A.F., Saakyan, I.Y. and Novikova, M.V. 1987. Gene pool of sources and donors for breeding winter bread wheat for immunity to bunt. Vaviliva, 176: 11-15.

Mamluk, O.F.M., Al Ahmed, and Makki, M.A. (1990). Current status of wheat diseases in Syria. Phytopath. Medit. 29: 143- 150.

Mamluk, O.F. and Zahour, A. (1993). Differential distribution and prevalence of Tilletia foetida (Walt.) Liro and T. caries (DC) Tul. On bread wheat and durum wheat. Phytopathology Medit. 32: 25- 32.

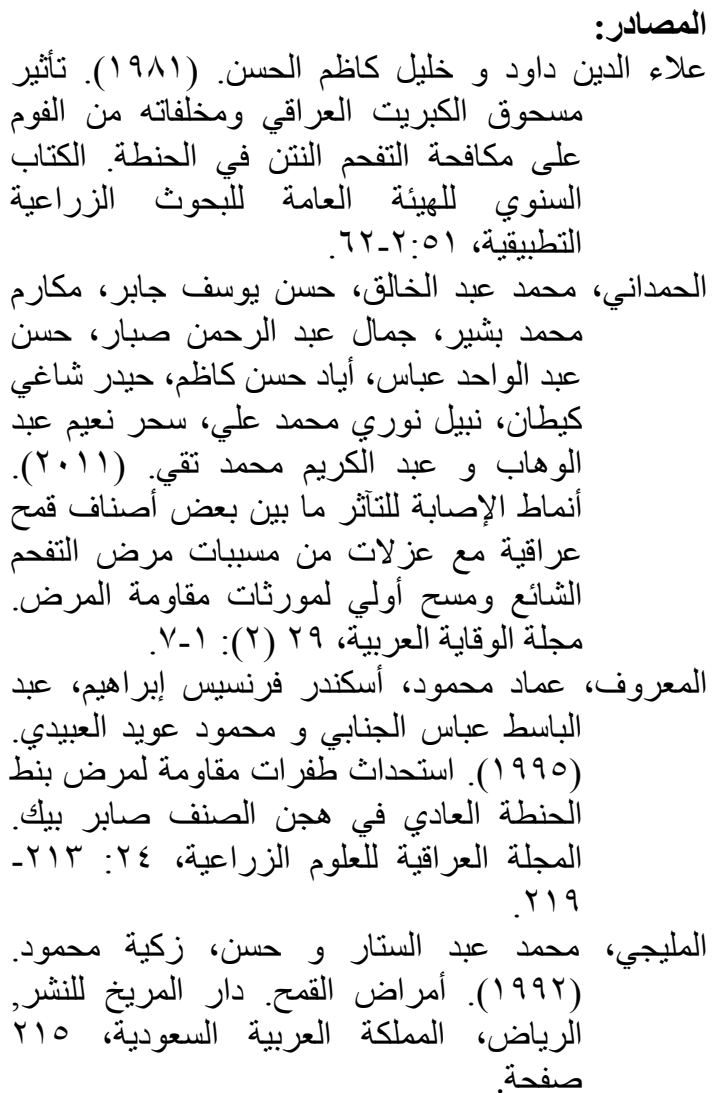

Al-Baldawi, A.A. (1993). Occurrence and importance of wheat and barley diseases in Iraq. Pages 105-113 In: Proc. Workshop on the Technology Transfer in the Production of Cereals and Legumes, September 20-22, 1993, Mosul, Iraq.

AL- Maroof, E.M., Faidh, F.A. and Queli. A.I. (2004). Efficiency of some fungicides in common bunt disease control in wheat. Page 329-336 In: Proc. $2^{\text {nd }}$ Int. Conf. Of Development and the Environment in the Arab World, March, 23-25.

Bonde, M.R., Prescott, J.M., Matsumoto, T.T. \& Peterson, G.L. (1987). Possible dissemination of teliospores of Tilletia indica by the practice of burning wheat stubble. Phytopathology, 77: 639 (abstr.).

Fischer, G.W. \& Holton, C.S. (1943). Studies of the susceptibility of forage grasses to cereal smut fungi IV. Cross-inoculation experiments with Urocystis tritici, U. occulta and U. agropyri. Phytopathology, 33: 910-921.

Gaudete, D.A. and Puchalski, B.L. (1989). Races of common bunt (Tilletia 
Varenitsa, E.T., Saakyan, 1.Y., Mozgovoi, A.F., Kochetygov, G.V. and Gradskov. S.M. (1987). Using derivatives of the variety Zarya as donors of resistance to bunt. Lenina, 4: 3-5.

Yarham, D. (1993). Soil borne spores as a source of inoculums for wheat bunt Plant Pathology. 42: 654-656.
Parlak, Y. (1981). Seed-borne pathogens on wheat (particularly smut). EPPO Bul. 11: 83- 86.

Souza, E., Windes, J.M., Sunderman, D.W., Whitmore, J., Kruk, M. \& Goates, B. (1995). Registration of 'Bonneville' hard red winter wheat. Crop Sci., 35: 1218-1219.

\section{Abstract \\ Detection of pathological changes in Tilletia spp. The causal agent of covered smut (Bunt) disease in Iraq}

Six isolates of Tilletia caries and T. foetida, the causal agents of wheat common bunt have been collected from six regions in Iraq and identified by their reaction to 10 differential wheat lines, each containing single bunt resistant gene. Diversity of the pathogen isolates was confirmed according to their reactions. BU6 from Al-Qayarah (Mosul) was the most virulent isolate which overcome all the resistant genes except $B t 7$ and $B t 10$. The least virulent isolates were BU1 and BU4. Bt7 ، Bt10 genes stayed resistant to all the isolates and the infection didn't exceed 10\%, although the isolates were mixture of Tilletia tritici and Tilletia laevis, followed by Bt5 which was resistant to most of the isolates except for BU5 and BU6 which excelled this resistance.

Key words: Common bunt of wheat. Iraqi isolates. Differential sets. 